\title{
Entanglement entropy in strongly correlated systems with confinement/deconfinement phase transition and anisotropy
}

\author{
Pavel Slepov ${ }^{1, *}$ \\ ${ }^{1}$ Steklov Mathematical Institute, Russian Academy of Sciences, \\ Gubkina str. 8, 119991, Moscow, Russia
}

\begin{abstract}
Five-dimensional anisotropic gravity with nontrivial dilaton field and two Maxwell fields is chosen for the holographic model, which allows to reproduce the multiplicity dependence on energy obtained from heavy-ions collisions $[1,2]$. Holographic entanglement entropy and its density are calculated for three-dimensional subsystems in this anisotropic background. These elongated subsystems have arbitrary spatial orientation in relation to the line of heavy-ions collisions. The divergences of holographic entanglement entropy are discussed. The entanglement entropy density has sharp spikes around the critical temperature for given chemical potential and anisotropy.
\end{abstract}

\section{Introduction}

One of the main experimental problems in high energy physics is the study of the QCD phase diagram [3]. Heavy-ions collisions (HIC) experiments at RHIC and LHC have provided solid evidence for a quark-gluon plasma (QGP) phase at high temperatures and densities $[4,5]$.

There are several theoretical approaches to describe the QCD phase diagram. The lattice calculations is a powerful method to describe phase transition, but it works for very small chemical potentials, because the lattice approach has the sign problem for $\mu>0$ [6]. The gravity/gauge duality [7] is an alternative method to study the QCD phase diagram [8-11]. One expects that near the first-order phase transition entanglement entropy has not a smooth behaviour. This is a reason to study HEE (holographic entanglement entropy) for holographic models of QCD.

There are several experimental and theoretical indications that QGP created in HIC has anisotropy right after the collision $[12,13]$. The isotropisation occurs at about $1 \mathrm{fm} / \mathrm{c}$ [13]. As a holographic anisotropy model, five-dimensional anisotropic gravity with nontrivial dilaton field and two Maxwell fields is chosen [1]. The anisotropy parameter is chosen to reproduce the multiplicity dependence on energy obtained from heavy-ions collisions [12].

The entanglement entropy depends on the orientation of the area in respect to the axes of the collisions. One can consider the influence of anisotropy on the HEE and its thermalization in the case of zero [14] and non-zero chemical potential [15]. In this talk the results [16] of HEE calculations for arbitrary oriented subsystems for the model [1] are presented. Note that the HEE has been considered in other models in papers [17-21].

\footnotetext{
*e-mail: slepov@mi-ras.ru
} 


\section{The model}

The gravitational theory dual to the anisotropic field theory is considered. This gravitational theory is defined by the Einstein-Dilaton-two-Maxwell action with potential $V$ for the dilaton field $\phi$ and functions $f_{1}$ and $f_{2}$ for two Maxwell fields [1]:

$$
S=\frac{1}{16 \pi G_{5}} \int d^{5} x \sqrt{-g}\left[R-\frac{f_{1}(\phi)}{4} F_{(1)}^{2}-\frac{f_{2}(\phi)}{4} F_{(2)}^{2}-\frac{1}{2}(\partial \phi)^{2}-V(\phi)\right],
$$

where $F_{(1)}^{2}$ and $F_{(2)}^{2}$ are the squares of the Maxwell fields.

The ansatz with the Maxwell fields $F_{(1) \mu v}=\partial_{\mu} A_{(1) v}-\partial_{v} A_{(1) \mu}, A_{(1) \mu}=A_{t}(z) \delta_{\mu}^{0}, F_{(2)}=$ $q d y^{1} \wedge d y^{2}, \phi=\phi(z)$ and metric

$$
d s^{2}=\frac{L^{2} b(z)}{z^{2}}\left[-g(z) d t^{2}+d x^{2}+\left(\frac{z}{L}\right)^{2-\frac{2}{v}}\left(d y_{1}^{2}+d y_{2}^{2}\right)+\frac{d z^{2}}{g(z)}\right], \quad b(z)=\mathrm{e}^{c z^{2} / 2},
$$

where $g(z)$ is the blackening function, $b(z)$ is the warp factor and $L$ is the characteristic geometry length scale and we set $L=1$. The advantage of this model is that we can find functions $V, f_{1}, f_{2}$ and $g$ explicitly. It is noteworthy, that the dilaton potential in this model can be approximated by the sum of two exponents. An explicit isotropic solution for the dilaton potential as a sum of two exponents and zero chemical potential has been constructed in [22]. It would be interesting to generalize this construction to the anisotropic and non-zero chemical potential cases.

The thermodynamical properties of the anisotropic holographic model were studied in [1]. The temperature of the model is given as

$$
T=\left|\frac{g^{\prime}\left(z_{h}\right)}{4 \pi}\right|=\frac{e^{-\frac{3 c_{h}^{2}}{4}}}{2 \pi z_{h}}\left|\frac{1}{\mathfrak{5}\left(\frac{3}{4} c z_{h}^{2}\right)}+\frac{\mu^{2} c z_{h}^{2+\frac{2}{v}} e^{\frac{c z_{h}^{2}}{4}}}{4\left(1-e^{\frac{c z_{h}^{2}}{4}}\right)^{2}}\left(1-e^{\frac{c z_{h}^{2}}{4}} \frac{\mathfrak{5}\left(c z_{h}^{2}\right)}{\mathfrak{5}\left(\frac{3}{4} c z_{h}^{2}\right)}\right)\right|,
$$

where for the function $(5)$ the expression could be presented:

$$
\mathfrak{b}(x)=\sum_{n=0}^{\infty} \frac{(-1)^{n} x^{n}}{n !\left(1+n+\frac{1}{v}\right)} .
$$

The expressions for thermal entropy and the free energy are the following:

$$
s=\frac{e^{\frac{3}{4} c z_{h}^{2}}}{4} z_{h}^{-\frac{(v+2)}{v}}, \quad F\left(z_{h}\right)=\int s d T=\int_{z_{h}}^{z_{h_{2}}} s \frac{d T}{d z_{h}} d z_{h},
$$

where $z_{h_{2}}$ is the second horizon (at $z_{h_{2}}$ the temperature equals to zero ).

The function $T\left(z_{h}\right)$ at $0<\mu<\mu_{c r}(c, v)$ is three-valued, and the free energy shows the swallow-tailed dependence on the temperature in this range of chemical potentials (Fig.1A). The lowest values of the free energy corresponds to the thermodanymically stable phases. The free energy $F\left(z_{h}\right)$ intersections with the horizontal axis give the values of the HawkingPage horizons (Fig.1B). Note that the black hole solution is always dominant with respect to thermal AdS for $\mu \neq 0$.

For $0<\mu<\mu_{c r}(c, v)$ the dependence of the free energy on the temperature looks like the swallow-tailed shape both in isotropic and anisotropic cases. A large black hole transits to a small one (BB phase transition) at $T=T_{B B}(\mu)$ (Fig.2A). Note that the thermal entropy has 
significant fluctuations near the BB phase transition points (Fig.2B). With the increment of the chemical potential $\mu$ from zero to $\mu_{c r}$, the loop of the swallow-tailed shape shrinks at $\mu=$ $\mu_{c r}(c, v)$. For $\mu>\mu_{c r}(c, v)$, the curve of the free energy increases smoothly with increasing temperature. The phase diagrams on the $(T, \mu)$-plane for this model were obtained in $[1,23]$, where differently oriented temporal Wilson loops were studied, see [24] for corresponding string tensions. The spatial Wilson loops for this model were studied in [25].

\section{Holographic entanglement entropy}

\subsection{Born-Infeld (BI) type action}

For HEE calculations let us consider general type of action [26]:

$$
\mathcal{S}=\frac{T}{2 \pi \alpha} \int_{-\ell / 2}^{\ell / 2} M(z(\xi)) \sqrt{\mathcal{F}(z(\xi))+\left(z^{\prime}(\xi)\right)^{2}} d \xi
$$
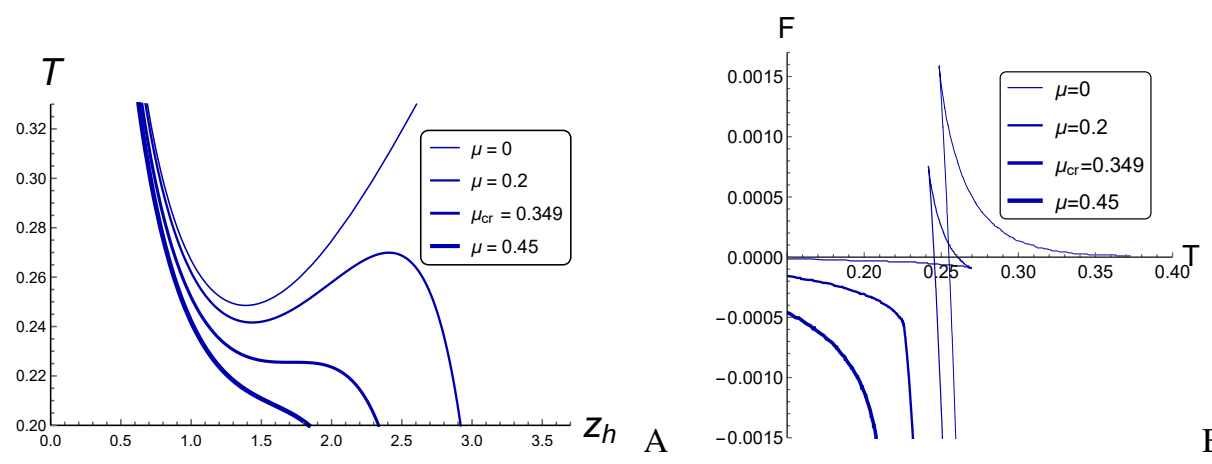

Figure 1. A) The temperature $T$ dependence on $z_{h}$ for $c=-1, v=4.5$ and different $\mu$ B) The free energy $F(T)$ for $c=-1, v=4.5$ and different $\mu$.
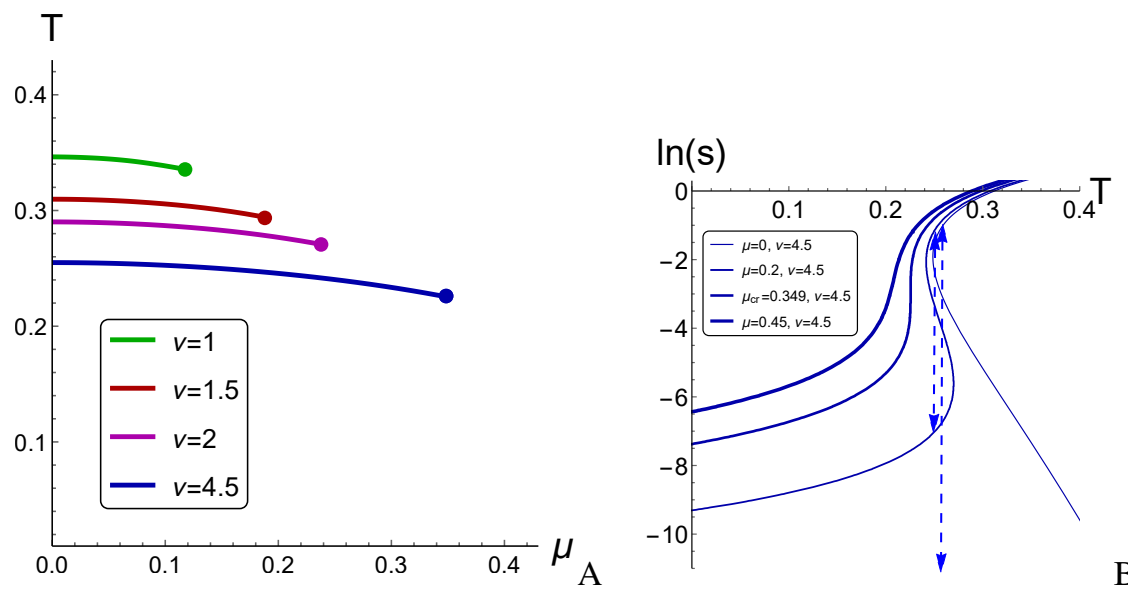

Figure 2. A) The BB phase transition on the $(T, \mu)$-plane for $c=-1$ and different $v$ B) The thermal entropy $s(T)$ for $c=-1, v=4.5$ and different $\mu$. 
The effective potential for this action can be introduced:

$$
\mathcal{V}(z(\xi)) \equiv M(z(\xi)) \sqrt{\mathcal{F}(z(\xi))} .
$$

We can express $z^{\prime}$ from the first integral and get representations for character length of the string and the action (the point $z_{*}$ is where $z^{\prime}(\xi)=0$ ):

$$
\frac{\ell}{2}=\int_{0}^{z_{*}} \frac{1}{\sqrt{\mathcal{F}(z)}} \frac{\mathcal{V}\left(z_{*}\right)}{\mathcal{V}(z)} \frac{d z}{\sqrt{1-\frac{V^{2}\left(z_{*}\right)}{V^{2}(z)}}}, \quad \frac{\mathcal{S}}{2}=\int_{\epsilon}^{z_{*}} \frac{M(z) d z}{\sqrt{1-\frac{V^{2}\left(z_{*}\right)}{V^{2}(z)}}} .
$$

In the case of HEE calculations function $M(z)$ has singular behaviour near $z \sim 0$ and near the horizon $z \sim z_{h}$. Function $\mathcal{F}(z)$ is finite near $z \sim 0$ and equals zero at the horizon. Potential $\mathcal{V}(z)$ is given by a decreasing function in the domain $0<z<z_{*}$ (Fig.3). We see that for decreasing potential the behaviour of the integrand (8) near $z \sim 0$ is defined by behavior of $M(z)$ near $z=0$, i.e. to study UV divergences we have to study asymptotic of $M(z)$ near $z=0$.

It is necessary to study the asymptotic of $\mathcal{S}$ for large $\ell$. There are two options for HEE to have $\ell \rightarrow \infty$, compare with consideration in [25] and refs. therein:

- The existence of a stationary point of $\mathcal{V}(z)$, i.e. $\left.\mathcal{V}^{\prime}(z)\right|_{z=z_{D W}}=0$, one calls this point a dynamical wall (DW) point. In this case one takes the top point $z_{*}$ equal to $z_{D W}$ and one gets

$$
\ell \underset{z \rightarrow z_{*}}{\sim} \frac{1}{\sqrt{\mathcal{F}\left(z_{D W}\right)}} \sqrt{\frac{\mathcal{V}\left(z_{D W}\right)}{\mathcal{V}^{\prime \prime}\left(z_{D W}\right)}} \log \left(z-z_{*}\right), \quad \mathcal{S} \underset{z \rightarrow z_{*}}{\sim} M\left(z_{D W}\right) \sqrt{\frac{\mathcal{V}\left(z_{D W}\right)}{\mathcal{V}^{\prime \prime}\left(z_{D W}\right)}} \log \left(z-z_{*}\right) .
$$

Hence $\mathcal{S} \sim \sigma_{D W} \cdot \ell$ and $\sigma_{D W}=M\left(z_{D W}\right) \sqrt{\mathcal{F}\left(z_{D W}\right)}$.

- If the stationary point $\mathcal{V}(z)$ is nonexistent in the domain $0<z<z_{h}$ it is still possible to get the behaviour $\ell \rightarrow \infty$. In this case it is supposed that near the horizon:

$$
\mathcal{F}(z)=f\left(z_{h}\right) \cdot\left(z_{h}-z\right)+O\left(\left(z_{h}-z\right)^{2}\right),
$$
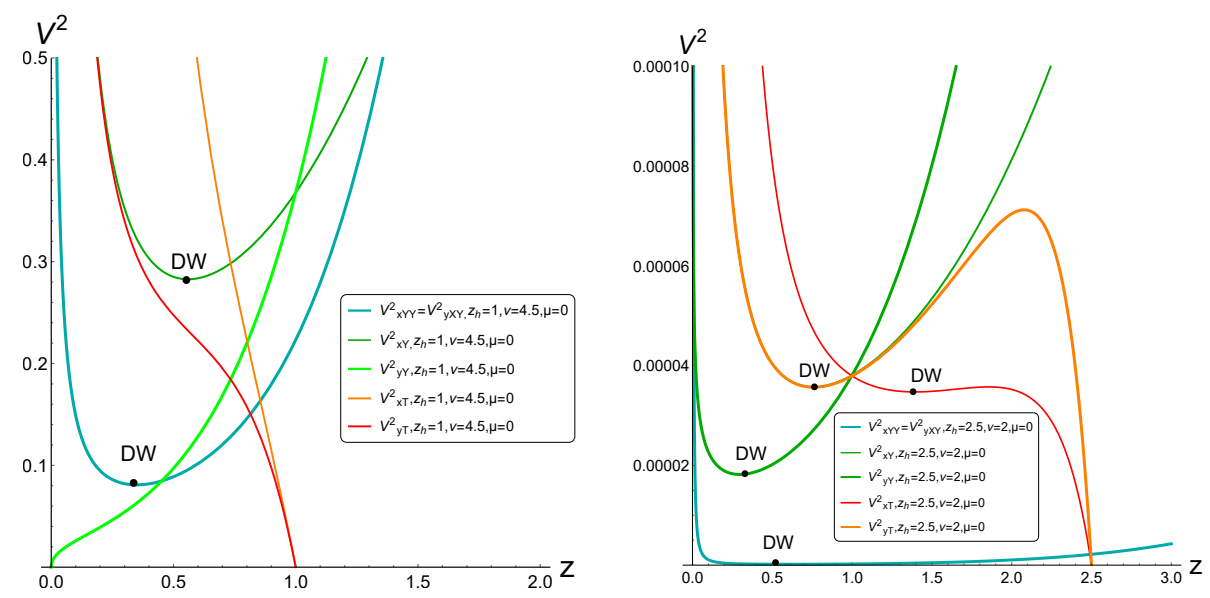

Figure 3. The comparison of the effective potentials $\mathcal{V}^{2}(z)$ for the differently oriented spatial, temporal Wilson loops and HEE calculated in model [1] for $\mu=0, c=-1$, different $z_{h}$ and $v$. 
So we take $z_{*}=z_{h}$. If $M(z) \underset{z \rightarrow z_{h}}{\rightarrow} \infty$ as $M(z) \underset{z \sim z_{h}}{\sim} \frac{\mathfrak{m}\left(z_{h}\right)}{\sqrt{z-z_{h}}}$ one gets

$$
\ell \underset{z \rightarrow z_{h}}{\sim} \frac{1}{\sqrt{f\left(z_{h}\right)}} \frac{1}{\sqrt{-\frac{2 \mathcal{V}^{\prime}\left(z_{h}\right)}{\mathcal{V}\left(z_{h}\right)}}} \log \left(z-z_{h}\right), \quad \mathcal{S} \underset{z \rightarrow z_{*}}{\sim} \mathrm{m}\left(z_{h}\right) \frac{1}{\sqrt{-\frac{2 \mathcal{V}^{\prime}\left(z_{h}\right)}{\mathcal{V}\left(z_{h}\right)}}} \log \left(z-z_{h}\right),
$$

and the tension is $\sigma_{h}=\mathfrak{m}\left(z_{h}\right) \sqrt{f\left(z_{h}\right)}$.

\subsection{General framework}

Let us deal with the system that consist of two spatially disjoint parts $A$ and $\bar{A}$ state $\Psi$ and a density matrix for subsystem $A$. Entanglement entropy is defined as the von Neumann entropy for reduced density matrix:

$$
S(A)=-\operatorname{tr}_{A} \rho_{A} \log , \quad \rho_{A}=\operatorname{tr}_{\bar{A}}|\Psi\rangle\langle\Psi| .
$$

The calculations of the entanglement entropy appears to be a sophisticated problem. However, one can compute its holographic dual. The HEE of a subsystem $A$ is given by the formula $[27,28]$ :

$$
S=\frac{\mathcal{A}}{4 G_{5}}
$$

where $\mathcal{A}$ is the area of the minimal three-dimensional surface, the boundary of which coincides with the boundary of the region $A$. The area of the surface is defined by the relation:

$$
\mathcal{A}=\int d^{3} \sigma \sqrt{\left|\operatorname{det} g_{m n} \partial_{\alpha} X^{m} \partial_{\beta} X^{n}\right|}, \quad \sigma^{1}=x, \quad \sigma^{2}=y_{1}, \quad \sigma^{3}=y_{2} .
$$

We consider the regions $A$ that describe the two nuclei overlap area are of interest for this study (Fig.4) [15].
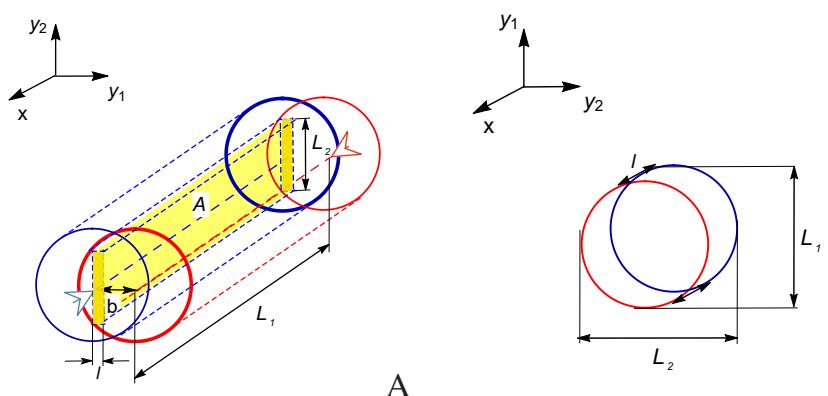

A

B

Figure 4. Schematic picture of HIC. Ions are presented as disks with diameter $D$ (blue and red disks). A) The overlapping area for a peripheral collision is approximated by the parallelepiped with sides $L_{1}, L_{2}$ and $\ell, L_{1}, L_{2}>>\ell \mathrm{B}$ ) The overlapping area is almost cylinder and the case of very short time after collision is considered, $\ell<<D, L_{1}, L_{2} \approx D$ 


\subsection{The form of the $\mathrm{BI}$ action for the entanglement entropy $S_{x Y Y}$ ( small subsystem in the longitudinal direction)}

First, consider the subsystem $A_{x Y Y}$ cut out along $x$-direction [15]:

$$
x \in\left[0,\left|l_{x}\right|<<L_{x}\right], \quad y_{1} \in\left[0, L_{y_{1}}\right], \quad y_{2} \in\left[0, L_{y_{2}}\right] .
$$

The minimal area surface is invariant under the $y_{1}$ and $y_{2}$ directions. The embedding function is the function of one coordinate only, $z=z(x)$. Taking into account (15) the HEE is the following:

$$
S_{x Y Y}=\frac{\mathcal{A}_{x Y Y}}{L_{y_{1}} L_{y_{2}}}=2 \int_{0}^{z_{*}} \frac{b_{s}^{3 / 2}(z)}{z^{1+2 / v}} \sqrt{1+\frac{\left(z^{\prime}\right)^{2}}{g(z)}} d x
$$

where

$$
b_{s}\left(z, z_{h}, v, c\right) \equiv e^{c z^{2} / 2+\sqrt{\frac{2}{3}} \phi\left(z, z_{h}, v, c\right)} .
$$

In term of the BI-action notations (6) we have:

$$
\mathcal{V}_{x Y Y}(z)=\frac{b_{s}^{3 / 2}(z)}{z^{1+2 / v}}, \quad M_{x Y Y}(z)=\frac{b_{s}^{3 / 2}(z)}{z^{1+2 / v} \sqrt{g(z)}}, \quad \mathcal{F}_{x Y Y}(z)=g(z) .
$$

Therefore, according (8) we have:

$$
\begin{aligned}
\frac{\ell_{x Y Y}}{2} & =\int_{0}^{z_{*}} \frac{1}{\sqrt{g(z)}}\left(\frac{z^{1+2 / v}}{z_{*}^{1+2 / v}}\right)\left(\frac{b_{s}\left(z_{*}\right)}{b_{s}(z)}\right)^{3 / 2} \frac{d z}{\sqrt{1-\frac{\mathcal{V}_{x Y}^{2}\left(z_{*}\right)}{\mathcal{V}_{x Y Y}^{2}(z)}}}, \\
\frac{\mathcal{S}_{x Y Y}}{2} & =\int_{\epsilon}^{z_{*}} \frac{b_{s}^{3 / 2}(z) d z}{\left.z^{1+2 / v} \sqrt{g(z)\left(1-\frac{\mathcal{V}_{x Y Y}^{2}(z)}{\mathcal{V}_{x Y Y}^{2}\left(z_{*}\right)}\right.}\right)} .
\end{aligned}
$$

The UV divergencies of HEE are defined by behaviour of $M_{x Y Y}$ at $z \sim 0$ given by (18) and taking into account that the asymptotics of $\phi$ is given by (eq. (2.58) in [1]). $M_{X Y Y}(z)$ has an integrable singularity at $z=0$ for $v>1.67$ :

$$
M_{x Y Y}(z) \underset{z \sim 0}{\sim} \frac{b_{s}^{3 / 2}(z)}{z^{1+2 / v}}=z^{\kappa_{X Y Y}} B_{s}^{3 / 2}\left(v, c, z_{h}\right), \quad \kappa_{x Y Y}(v)=\frac{\sqrt{6(v-1)}}{v}-1-2 / v .
$$

\subsection{The form of the BI action for the entanglement entropy $\mathcal{S}_{y X Y}$ ( small subsystem delineated along the transversal direction)}

Another possible subsystem $A$ can be divided along the $y_{1}$-direction (which is equivalent to dividing it along $\left.y_{2}\right)$ [15]. It is also assumed that $z=z\left(y_{1}\right)$ and

$$
x \in\left[0, L_{x}\right], \quad y_{1} \in\left[0,\left|l_{y_{1}}\right|<<L_{y_{1}}\right], \quad y_{2} \in\left[0, L_{y_{2}}\right] .
$$

Thus, the expression for the entanglement entropy is defined by

$$
\mathcal{S}_{y X Y}=\frac{\mathcal{L}_{y X Y}}{L_{x} L_{y 2}}=2 \int_{0}^{z_{*}} \frac{b_{s}^{3 / 2}(z)}{z^{1+2 / v}} \sqrt{1+\frac{\left(z^{\prime}\right)^{2}}{g(z) z^{2-2 / v}}} d x .
$$

In notation (6) we have:

$$
\mathcal{V}_{y X Y}(z)=\frac{b_{s}^{3 / 2}(z)}{z^{1+2 / v}}, \quad M_{y X Y}(z)=\frac{b_{s}^{3 / 2}(z)}{z^{2+1 / v} \sqrt{g(z)}}, \quad \mathcal{F}_{y X Y}(z)=g(z) z^{2-2 / v},
$$


and therefore it is possible to write the expressions for $\ell_{y X Y}$ and $\mathcal{S}_{y X Y}$ as in previous case. The function $M_{y X Y}(z)$ has a nonintegrable singularity at $z=0$ for all $v$ values at $z \sim 0$ :

$$
M_{y X Y}(z) \underset{z \sim 0}{\sim} \frac{b_{s}^{3 / 2}(z)}{z^{2+1 / v}}=B_{s}^{3 / 2}\left(v, c, z_{h}\right) z^{\kappa_{y X Y}}, \quad \kappa_{y X Y}(v) \equiv \frac{\sqrt{6(v-1)}}{v}-2-1 / v
$$

where $\kappa_{y X Y}(v)=\kappa_{x Y Y}(v)-1+1 / v$.
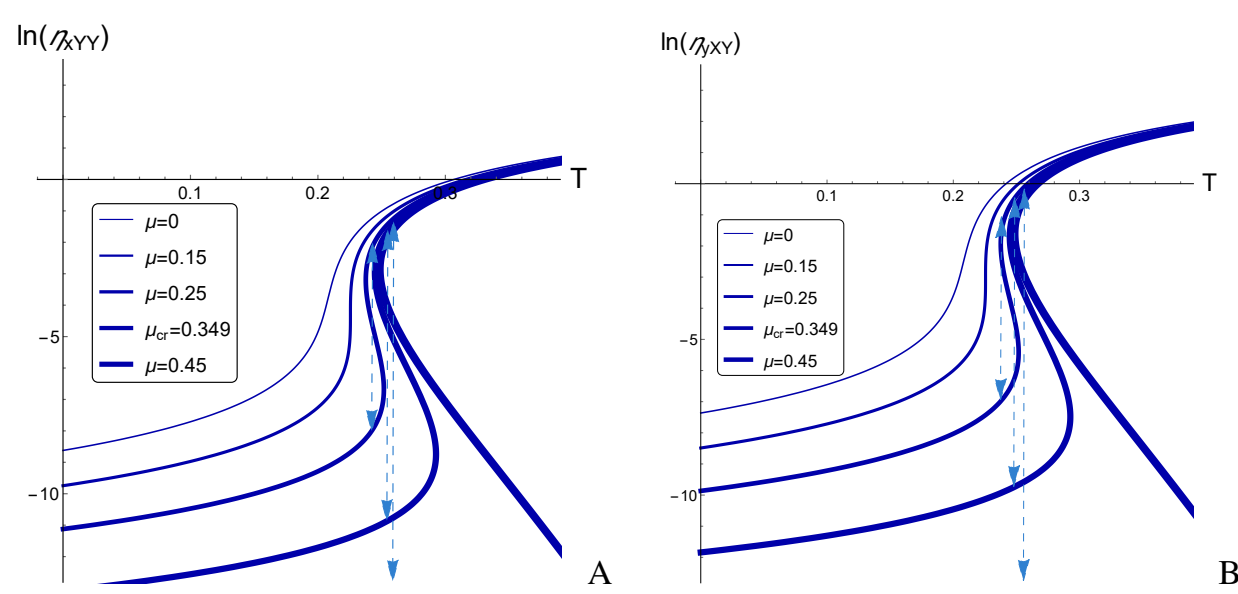

Figure 5. HEE density for A) $\mathrm{xYY}$ and $\mathrm{B}$ ) $\mathrm{yXY}$ orientations, $c=-1, v=4.5$ and different chemical potentials, $\ell=2$.

\subsection{Density of holographic entanglement entropy}

It is convenient to consider the entanglement entropy density which is not a divergent quantity. HEE density for fixed temperature $T$ (i.e. fixed $z_{h}$ ) can be defined in BI terms (6) by

$$
\eta\left(z_{*}\right)=\frac{d S\left(z_{*}\right)}{d \ell\left(z_{*}\right)}=\left.\frac{\frac{d S}{d z_{*}}}{\frac{d \ell}{d z_{*}}}\right|_{z=z_{*}}=\mathcal{V}\left(z_{*}\right)=\left.\frac{b_{s}^{3 / 2}(z)}{z^{1+2 / v}}\right|_{z=z_{*}},
$$

and we see that $\eta=\mathcal{V}_{x Y Y}\left(z_{*}\right)=\mathcal{V}_{y X Y}\left(z_{*}\right)$ and the form of entanglement entropy density does not depend on the orientation. But we can expect the angular dependence of the density due to the angular dependence of the characteristic length $\ell$ (Fig.5). Note that $\ell$ depends on $z_{*}$ and $z_{h}$ for fixed $\mu, v, c$ and is not easy task to fix the length.

\section{Conclusion}

HEE and its density were calculated in the holographic anisotropic model [1] supported by the Einstein-Dilaton-two-Maxwell action. HEE density has significant fluctuations near the BB phase transition temperature for fixed chemical potential and anisotropy. More detailed consideration of the HEE renormalizations, the cases of $\theta$ - oriented HEE and its density will be presented in [16]. We hope that these results and their interpretation could be of interest for experiments at the future facilities of RHIC, LHC, FAIR and NICA. 


\section{Acknowledgments}

This paper is based on a talk at the XXIV International Workshop High Energy Physics and Quantum Field Theory at Sochi, Russia on 22 - 29 September 2019. Author would like to thank the organizers of QFTHEP'2019 for the opportunity to give this report. Author would like to thank Prof. I.Ya. Aref'eva for attracting me to the research presented in the report and numerous discussions and also K.A. Rannu and A.V. Patrushev for useful discussions. This work was supported by RFBR Grant 18-02-40069.

\section{References}

[1] I. Aref'eva and K. Rannu, JHEP 1805, 206 (2018)

[2] I. Ya. Aref'eva, "Theoretical studies of heavy ion collisions", this proceedings.

[3] A. Andronic, P. Braun-Munzinger, K. Redlich and J. Stachel, Nature 561, no. 7723, 321 (2018)

[4] Adams J et al. (STAR Collab.) Nucl. Phys. A 757102 (2005)

[5] Adcox K et al. (PHENIX Collab.) Nucl. Phys. A 757184 (2005)

[6] G. Aarts, J. Phys. Conf. Ser. 706, 022004 (2016)

[7] J. M. Maldacena, Phys. Rev. Lett. 80, 4859 (1998)

[8] E. Witten, Adv.Theor.Math.Phys. 2, 505 (1998)

[9] J. Casalderrey-Solana, H. Liu, D. Mateos, K. Rajagopal and U. A. Wiedemann, Cambridge University Press, (2014)

[10] I. Ya. Aref'eva, Phys. Usp. 57527 (2014)

[11] O. DeWolfe, S. S. Gubser, C. Rosen and D. Teaney, Prog. Part. Nucl. Phys. 75, 86 (2014)

[12] I. Y. Aref'eva and A. A. Golubtsova, JHEP 1504, 011 (2015)

[13] M. Strickland, Pramana 84, 671 (2015).

[14] I. Y. Aref'eva, A. A. Golubtsova and E. Gourgoulhon, JHEP 1609, 142 (2016)

[15] I. Aref'eva, Phys.Part.Nucl.Lett.16 no.5. (2019)

[16] I. Aref'eva, A. Patrushev and P. Slepov, Holographic Entanglement Entropy in Anisotropic Background with Confinement-Deconfinement Phase Transition, in preparation.

[17] I. R. Klebanov, D. Kutasov and A. Murugan, Nucl. Phys. B 796 (2008) 274

[18] S. J. Zhang, Nucl. Phys. B 916, 304 (2017)

[19] J. Knaute and B. Kampfer, Phys. Rev. D 96, no. 10, 106003 (2017)

[20] M. Ali-Akbari and M. Lezgi, Phys. Rev. D 96, no. 8, 086014 (2017)

[21] D. Dudal and S. Mahapatra, JHEP 1807, 120 (2018)

[22] I. Ya. Aref 'eva, A. A. Golubtsova and G. Policastro, JHEP 1609, 117 (2019)

[23] I. Aref'eva, K. Rannu and P. Slepov, Phys.Lett. B 792, 470 (2019)

[24] I. Aref'eva, K. Rannu, P.Slepov, "Cornell potential for anisotropic QGP with non-zero chemical potential", this proceedings.

[25] I. Aref'eva, EPJ Web Conf. 191, 05010 (2018).

[26] I. Ya. Aref'eva, EPJ Web Conf. 164, 01014 (2017)

[27] S. Ryu and T. Takayanagi, Phys. Rev. Lett. 96, 181602 (2006)

[28] V. E. Hubeny, M. Rangamani and T. Takayanagi, JHEP 0707, 062 (2007) 